\title{
Learning while multitasking: Short and long-term benefits of brain
}

stimulation

Frank, B. ${ }^{1 *}$, Harty, S. ${ }^{2}$, Kluge, A. ${ }^{1}, \&$ Cohen Kadosh, R..$^{2 *}$

${ }^{1}$ Ruhr-University Bochum, Department of Work, Organisational and Business

Psychology, Bochum, Germany

${ }^{2}$ University of Oxford, Department of Experimental Psychology, Oxford, United Kingdom

* corresponding authors: barbara.frank@ rub.de, roi.cohenkadosh@psy.ox.ac.uk 


\section{Learning while multitasking: Short and long-term benefits of brain stimulation}

We employed a simulated production task that mimics the real-world skill acquisition required of operators working in control rooms of power plants to assess short and long-term effects of transcranial random noise stimulation (tRNS). tRNS has shown potential for enhancing learning and performance of cognitive skills. 40 subjects ( 24 female) learned how to execute the simulated production task during the training phase and were required to perform a secondary task during the skill acquisition phase, while they received active (12 minutes) or sham tRNS on DLPFC. After two weeks they had to recall the task again without any stimulation. The results demonstrate that tRNS promoted better multitasking as reflected by better performance in a secondary task during and immediately after tRNS. However, two weeks later, beneficial effect of tRNS on retention was moderated by general mental ability. Particularly, tRNS benefited those with lower general mental ability.

Practitioner summary: By using a simulated production task, we assessed the effects of tRNS on learning and skill retention. The study indicates that neurostimulation can enhance learning of multiple complex tasks. Moreover, it shows that retention of those tasks can be supported by neurostimulation, especially for those with lower general mental ability.

Keywords: tRNS, brain stimulation, cognitive enhancement, complex task, dualtask

\section{$1 \quad$ Introduction}

In many scenarios initially learned skills are not used immediately or are used infrequently after learning. After a period of disuse it can be challenging to retain those new skills because the lack of retrieval activities, probably due to a loss of retrieval strength (Arthur et al. 2013). This can be traced back to the New Theory of Disuse, which states that high storage strength and high retrieval strength are important for the retention of skills (Bjork and Bjork 2006, 1992); wherein the former describes how well 
information is acquired and stored in memory, while the latter describes the extent to which stored information is accessible.

In work places like aviation, military, or process industry it is critical to retain learned skills over periods of disuse to prevent incidents and accidents. To date, existing methods for improving skill retention focus on refresher interventions with practical repetitions or tests and overlearning (Kluge and Frank 2014, McDaniel, Brown, and Roediger 2014, Driskell, Willis, and Copper 1992). However, current technological developments pave the way for new methods to enhance skill acquisition. In particular, the field of neuroergonomics, the integration of neuroscience and ergonomics, holds promise for fostering innovations that will lead to more efficient and safer work conditions (Parasuraman 2003). In this respect, the use of brain stimulation, which has been suggested to modulate neuroplasticity, is a promising approach for improving skill retention. Transcranial electrical stimulation (tES) is one tool that holds particular promise in this area. For instance, several studies have shown that $\mathrm{tES}$ is associated with improvements in performance after the learning of numerical skills, language, motor tasks and complex tasks (for reviews, Krause and Cohen Kadosh 2013; Schroeder et al. 2017; Simonsmeier et al. 2018; Santarnecchi et al. 2015). Moreover, a recent metaanalysis has found promising results on using transcranial electric stimulation for enhancing learning and retention (Simonsmeier et al. 2018).

Transcranial random noise stimulation (tRNS) is a variant of tES that has only been employed in a limited number of studies to date, but emerging evidence suggests that tRNS produces greater cortical excitability, neuroplastic change, and learning effects compared to more well-known tES methods (Fertonani, Pirulli, and Miniussi 2011, Terney et al. 2008). With tRNS, the intensity and the frequency of the alternating current vary in a randomized manner within specified ranges. The effects of tRNS, at 
least when applied within the 1-2 mA range, are believed to be excitatory at both electrode sites due to its fast-oscillating field that putatively depolarizes neurons irrespective of the polarity of current flow (Terney et al. 2008). It can accordingly be bilaterally applied to the dorsolateral prefrontal cortex (DLPFC) to increase excitability simultaneously in both left and right DLPFC regions (Snowball et al. 2013), which are known to be important for learning and memory formation (Anderson 2005, Chein and Schneider 2012). tRNS has been shown to modulate neural excitability and neuroplasticity when applied over the DLPFC to support retention over mid-term and long-term periods (Snowball et al. 2013, Cappelletti et al. 2013). Previous studies have suggested that one of the mechanisms through which tRNS may achieve its functional effects is stochastic facilitation (Fertonani and Miniussi 2016, van der Groen and Wenderoth 2016). Stochastic facilitation is the term used to describe the general phenomenon whereby adding an appropriate level of random noise to non-linear systems can enhance the output of subthreshold signals (McDonnell and Ward 2011).

\subsection{The Current Study}

In the current study, we sought to apply this knowledge and reasoning to the context of skill acquisition in a simulated real-world task. For handling non-routine situations, operators learn and use "standard operating procedures" (SOP, Kluge 2014). SOPs are predefined procedures that describe how and what the operator has to do on upcoming occasions (Wickens and Hollands 2000). They constitute complex cognitive skills (van Merriënboer 1997). Operators have to recall what to do and how to execute the steps of the procedure at the right time and in the right sequence (e.g. interface interaction, timing, sequence of steps, rules). In many situations such SOPs include multitasking in the form of dual-tasks. Dual-tasks consists of two tasks that have to be performed in parallel and need to be synchronised in time (Proctor and Dutta 1995, Wickens 2008, 
Wickens and McCarley 2008). Handling dual-tasks successfully is cognitively demanding, but essential in different work places including the industry, aviation, maritime industry, and medical domains. Notably, while tRNS to the best of our knowledge, has not been applied during multitasking to date, other forms of neurostimulation examined the efficacy of neurostimulation in this domain (Filmer, Mattingley and Dux 2013; Hsu et al. 2017; Nelson et al. 2016).

In the present study we used the start-up procedure of simulated waste water treatment, which includes a dual-task component, namely, the start-up procedure of simulated waste water treatment. This task, originally developed by engineers who are specialised in process control engineering, is a validated computer-based simulation of a complex control task characterised by high dynamics, interrelatedness, feedback and opaqueness (Burkolter et al. 2009). A key advantage of this task is that it resembles an ecological setting where learning takes place, such as in a factory.

Not surprisingly, previous studies have shown that subjects' performance, including performance on this task, is affected by general mental ability (Frank and Kluge 2018, Diamond 2013). Of note there is also evidence to suggest that the effects of tES may be sensitive to general mental ability and cognitive load. More specifically, it has been shown that the effects of tES are more pronounced in individuals with lower cognitive abilities (Santarnecchi et al. 2015, Sarkar, Dowker, and Cohen Kadosh 2014, Looi et al. 2016, Tseng and al. 2012). We therefore assessed general mental ability using the Wonderlic Personnel Test, which has been found to correlate with Wechsler Adult Intelligence Scale in previous studies (e.g. Wonderlic 2002, Hawkins et al. 1990). From the perspective of learning theories (e.g. (Bjork and Bjork 2006, 1992), the enhanced neural excitability induced by tRNS during skill acquisition may impact storage strength, and, therefore, skill retention. 
In line with previous findings, which reported an effect of tRNS on learning of complex tasks, we hypothesised that: 1) subjects who receive tRNS will show a higher performance in the learned dual-task in week 1. It was also of interest to determine the longevity of the tRNS effects; for this appraisal we hypothesised that 2) subjects who receive tRNS will show a retention effect after two weeks. While the long-term effect of tRNS have mainly been assessed after multi-session cognitive training (for a review, see Simonsmeier et al. 2018), here, we examined whether a single-session of tRNS applied for 12 minutes during skill acquisition will lead to higher performance after two weeks. Further, as mentioned above, we also hypothesised that 3) the tRNS-related improvements would depend on baseline general mental ability, as assessed using the Wonderlic Personnel Test.

\section{Method}

\subsection{Sample}

Forty students (24 female) from the University of Oxford took part in the present study. Twenty of them constituted the active group (13 females, mean age $=21.05, S D=0.80)$ and the other 20 constituted the sham group ( 11 females, mean age=23.58, $S D=1.00$; procedures for the sham group are described below). The groups did not differ in sex and age (all ps>.05). All subjects had normal or corrected-to-normal vision and no history of neurological or psychiatric illness. The subjects were recruited by postings on social networking sites and flyers distributed at the University of Oxford. Subjects received $£ 25$ for taking part in the study, and the study was approved by the Medical Sciences Interdivisional Research Ethics Committee at the University of Oxford. All subjects were novices in learning the complex task used in the study. 


\subsection{The Waste Water Treatment Simulation Task}

Main task: The subjects learned how to operate the microworld Wastewater Treatment Simulation (WaTrSim, Figure 1), which requires the application of a prototypical complex cognitive skill. The operation includes the start-up of the plant, consisting of 13 steps, which is assumed to be a non-routine task (Wickens and Hollands 2000). In WaTrSim, the operator's task is to separate waste water into fresh water and gas by starting up, controlling and monitoring the plant. The operation goal is to maximise the amount of purified water and gas and to minimise the amount of waste water. This goal is achieved by following a start-up procedure and considering the timing of actions. The start-up procedure is described in detail in Table 1. Performing the WaTrSim start-up procedure correctly and in a timely manner leads to a production outcome of a minimum of 200 litres of purified gas. The amount of purified gas produced during skill acquisition was used as a criterion for screening subjects for inclusion: The subjects were required to produce $>=200$ litres of purified gas. If they failed to produce this amount, they were excluded from participating in the study. The start-up procedure had to be completed in 180 seconds. The production of $>=200$ litres of purified gas depends on the correct and fast execution of the procedure. The primary performance measure was therefore production outcome of purified gas (Burkolter et al. 2009, Frank and Kluge 2017).

Secondary task: To induce workload and to mimic a realistic work setting, the subjects were required to perform a secondary task in addition to the main task. The secondary task is performed by monitoring the tank level of tank Ba every 50 seconds (Table 1 and Figure 1; tank Ba can be found top right). The performance measure in this case is the frequency (0-3) of monitoring tank level (Burkolter et al. 2009).

Subjects were told that their objective is to perform main and secondary task in parallel. A video of main and secondary tasks is provided as supplementary material. 


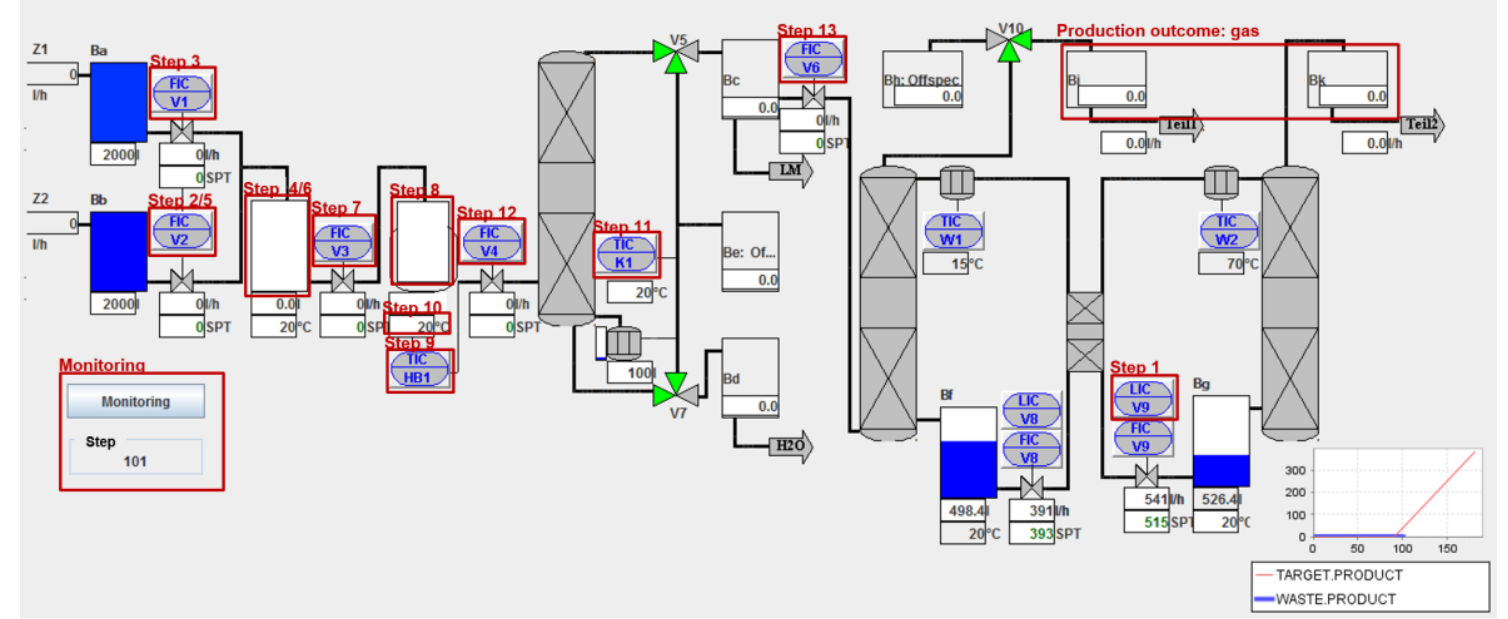

Figure 1. WaTrSim interface with valves (V1-V9), heaters (HB1, K1, W1, W2) and tanks (Ba, Bb, R1, HB1, Bc, Be, Bd, Bh, Bj, Bk, Bf, Bg). The main task is indicated by red boxes with labels "step 1-13" and secondary task is indicated by label "monitoring". See also supplementary video of main and secondary task

- Please include Table 1 here -

\subsection{Brain Stimulation}

Subjects received active or sham tRNS while performing the dual-task during the skill acquisition phase. Two circular electrodes $\left(25 \mathrm{~cm}^{2}\right)$ were positioned over areas of scalp corresponding to the DLPFC (F3 and F4 identified in accordance with the international 10-20 system for EEG electrode placement). Electrodes were encased in saline-soaked synthetic sponges to improve contact with the scalp and avoid skin irritation. Stimulation was delivered by a Starstim device (Starstim, Neuroelectrics). Noise in the high-frequency band $(100-500 \mathrm{~Hz})$ was applied as tRNS using lowfrequency band $(.1-100 \mathrm{~Hz})$ has been shown to be less effective (Terney et al. 2008, Fertonani, Pirulli, and Miniussi 2011).

For the active group, current of $1 \mathrm{~mA}$ was administered for 12 minutes, with 30 seconds ramp-up and 20 seconds ramp-down at the beginning and end, respectively. In the sham group the stimulation parameters were the same as for the active group with 
the exception that the stimulation was applied for only 10 seconds as opposed to 12 minutes. The stimulation was applied in a double-blind manner.

\subsection{Procedure}

The subjects attended two sessions (Figure 2). During skill acquisition subjects were trained on how to operate WaTrSim using the start-up procedure (120 minutes). At the beginning of the first session, general mental ability was measured using Wonderlic Personnel Test. Subjects answered 50 items in twelve minutes, including analogies, analysis of geometric figures, logic tasks, mathematical tasks, similarities or word definitions like "a boy is five years old and his sister is twice his age. When the boy is eight, how old will his sister be?" Correct answers were summed up (range 0-50). To take into account the possible effect of sleepiness on learning we measured the level of sleepiness using the Stanford sleepiness scale (Hoddes et al. 1973). Following this, subjects explored and familiarised themselves with WaTrSim twice. They were then given information and instructions about the start-up procedure (main task) and how to monitor the tank level of tank Ba while operating the start-up procedure (secondary task). Followed by four minutes resting phase. In a 12 minutes training session, subjects executed the dual-tasks with the help of a manual (3 minutes per each execution), while receiving active or sham tRNS. To allow for a better understanding of the potential temporal dynamics of tRNS effects, we divided this training period into two parts of 6 minutes each (first 6 min: Training 1; last 6 min: Training 2). After the completion of the training session, subjects had to operate WaTrSim three times (Test 1) without the manual and tRNS. Subjects were instructed to produce 200 litres of purified gas

minimum once. As mentioned above, production of this minimum quantity constituted a requisite criterion for inclusion. At the end, the subjects completed the Stanford Sleepiness Scale a second time and were asked in a short questionnaire to guess in 
which group (stimulation/no stimulation) they participated in order to assess the effectiveness of the blinding procedure.

To evaluate whether there was evidence of long-term tRNS effects, subjects returned to the lab two weeks after their initial training. During this second visit, they filled in the Stanford Sleepiness Scale and had to execute the dual-task twice without instruction from the experimenter, without the help of the manual, and without tRNS (Test 2; Figure 2)

Although the results are not reported here, electroencephalography was recorded from all individuals pre- and post- tRNS during skill acquisition and recall.

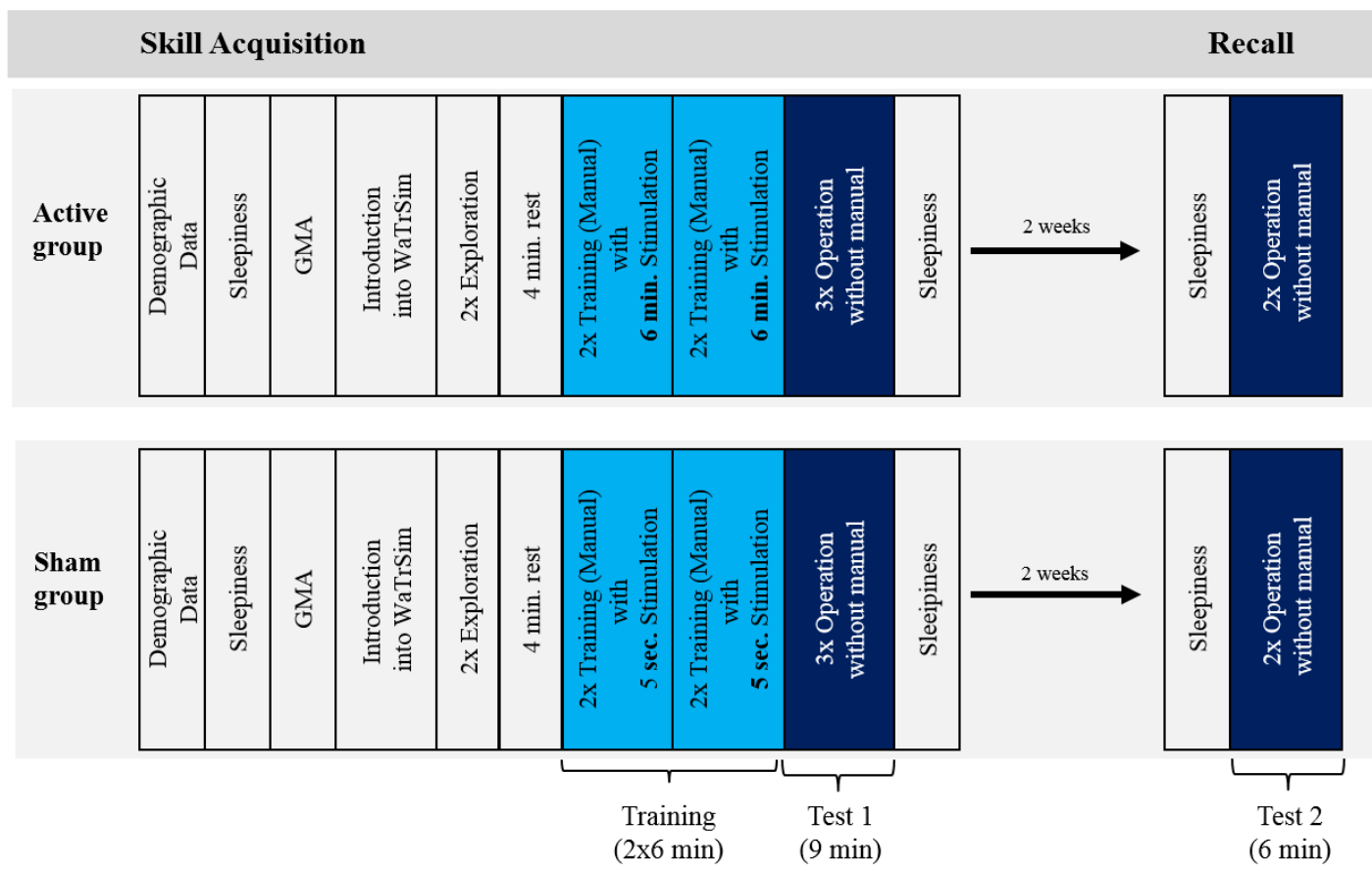

Figure 2. Procedure of active group (12 minutes tRNS) and sham group (10 seconds tRNS). One WaTrSim execution lasted for 3 minutes; GMA=General Mental Ability

\subsection{Variables and measures}

Stimulation (active vs. sham) was used as the independent variable. The dependent variables were production outcome (main task) and monitoring tank level (secondary task). These were measured at skill acquisition and two weeks later at skill recall 
- Production outcome: The amount of purified gas produced.

- Monitoring tank level: The number of times the level of tank Ba was checked $(0-3)$.

Performance of both groups was measured for the first six minutes of training (Training 1), the latter six minutes of training (Training 2) and for nine minutes of Test 1 at skill acquisition. After two weeks, performance was measured for six minutes of Test 2 at the recall phase.

General mental ability was controlled for with the Wonderlic Personnel Test (Wonderlic 2002) and sleepiness was assessed with Stanford Sleepiness Scale (Hoddes et al. 1973). For the production, outcome we applied a moderation analysis. This analysis is analogous with a general linear model that considers main effects and all interaction terms. For this analysis we used Hayes's (Hayes 2013) PROCESS module in SPSS and estimated the $95 \%$ bootstrapping (5,000 resamples) confidence intervals for the observed effects. We decomposed significant interactions to understand the source of the interaction using Aiken and West's recommendation. This is the standard procedure in moderation analysis, and entails examining the conditional effect one standard deviation above and below the mean (Aiken and West 1991). As the error residuals for monitoring were not normally distributed we used a non-parametric test (MannWhitney $U$ test) to examine the effect of stimulation. While we report the uncorrected pvalues, the results for the main analyses (production outcome and monitoring) were still significant when we corrected for multiple comparisons using Holm-Bonferroni sequential correction.

\section{$3 \quad$ Results}

The descriptive statistics are displayed in Table 2. One subject in the sham group was excluded due to not producing the requisite production outcome $>=200$ litres during 
skill acquisition. Subjects in the active and sham groups did not differ in guessing the type of stimulation they received at the end of skill acquisition $\left(\chi^{2}(1)=0.21, p=.651\right)$, suggesting that our blinding procedure was effective. Further, we can confirm that controlling for general mental ability showed no differences between both groups (Mann Whitney $U=152.5, n_{1}=20 n_{2}=19, p=.08$ ). As it is possible that performance on the main task would be compromised due to subjects' efforts to perform the secondary task, or vice versa, we assessed for evidence of this by running a correlation analysis. A trend toward a positive, rather than negative, correlation between production outcome and monitoring (Training 1: $r_{s}=.16, p=.33$; Training 2: $r_{s}=.24, p=.13$; Test 1: $r_{s}=.36$, $p=.025$; and Test 2: $\left.r_{s}=.56, p=.0002\right)$, indicated that performance on each of the tasks was not compromised by efforts to perform the other. The performance during training, Test 1 and Test 2 is given in Table 2 and Figure 3.

- Include Table 2 here -
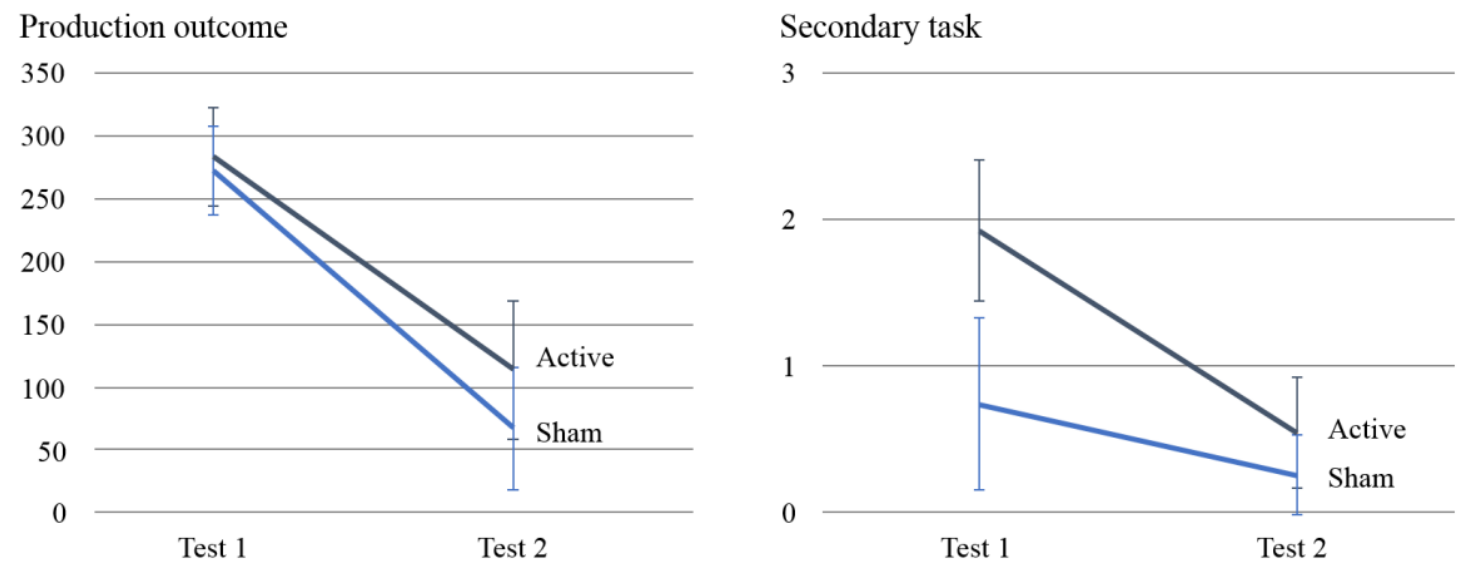

Figure 3. Performance of active and sham group in Test 1 and Test 2

\section{$\underline{\text { Skill acquisition }}$}

First, we tested whether both groups showed different performance levels at Training 1, Training 2, and Test 1 of skill acquisition. Production outcome was not affected by tRNS, or the interaction between tRNS and general mental ability at any phase (all $t$ 
values $<.86, p$ values $>$.4). However, production outcome was significantly related to general mental ability at all phases of performance (Training $1: \beta=.38, t(37)=2.3$, $p=.027$; Training $2: \beta=.47, t(37)=3.12, p=.004 ; \beta=.36$, Test $1: t(37)=2.17, p=.037)$.

In contrast, when we examined the effect of tRNS on the secondary task, we found that tRNS did improve the monitoring performance at each time points (Training 1: MannWhitney $U=68, n_{1}=20 n_{2}=19, \mathrm{p}=.0004$, effect size (r)=.61; Training 2: MannWhitney $U=76.5, n_{1}=20 n_{2}=19, \mathrm{p}=.001$, effect size=.55; Test 1: Mann-Whitney $U=$ 94, $n_{1}=20 n_{2}=19, p=.006$, effect size $\left.=.45\right)$.

\section{$\underline{\text { Retention }}$}

In the previous section the immediate effect of tRNS was assessed at Training 1 and 2, and Test 1 examined its short-lasting effect. To examine for long-lasting effects, we repeated the same analyses two weeks after the completion of the skill acquisition (Test 2). For production outcome, we found that there were no main effects of tRNS (whether subjects received active or sham tRNS two weeks earlier) or general mental ability (all t values $<1.14$, all $\mathrm{p}$ values $>.26$ ), but the interaction between them was significant $(F(1,35)=9.98, p=.003$, Cohen's d=.49, Figure 4). In contrast, for monitoring performance, we did not observe an effect of stimulation (Mann-Whitney $U=$ 152.5, $n_{1}=20 n_{2}=19, p=.29$, effect size=.21).

When we decomposed the interaction between stimulation and general mental ability (Aiken and West 1991) to examine their effect on production outcome, we found that stimulation was beneficial to those with lower general mental ability (Mean ${ }_{\mathrm{tRNS}}$ minus Mean $_{\text {sham }}=148, S E=47: t(37)=-3.16, p=.003$, Cohen's d=-.67), but not for those with higher general mental ability, which, descriptively, yielded an opposite trend $\left(\right.$ Mean $_{\mathrm{tRNS}}$ minus Mean sham $_{\text {- }}$-71, $S E=50: t(37)=1.41, p=.16$, Cohen's d=.32). 


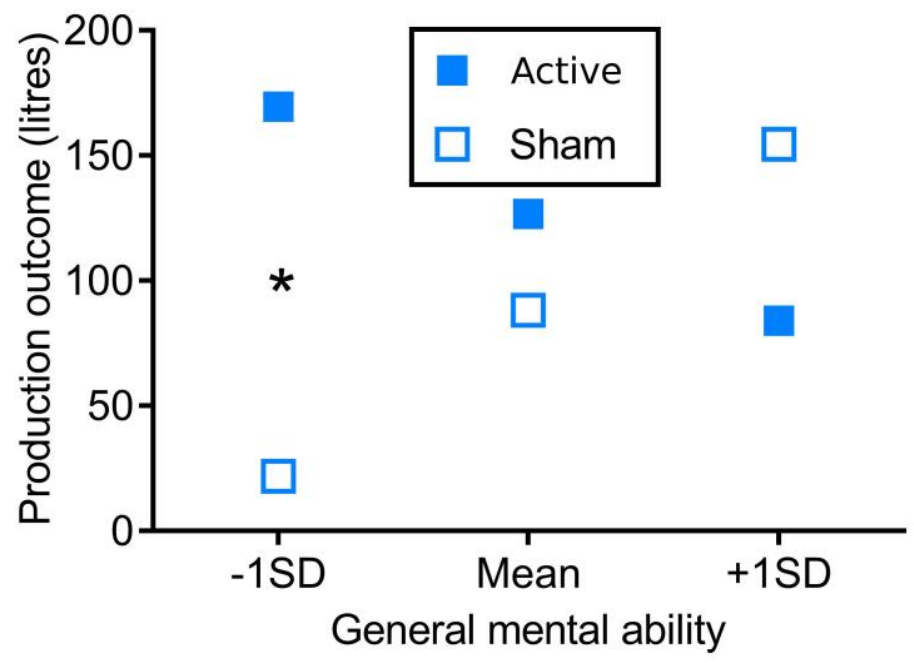

Figure 4. Production outcome (main task) at the recall stage was predicted by stimulation and general mental ability. tRNS was effective for those with lower general mental ability, showing a long-term effect of learning (retention), 2 weeks after skill acquisition. ${ }^{*} \mathrm{p}=.003$

\section{Discussion}

The aim of the present study was to test the immediate, short-term, and longterm effects of tRNS on skill acquisition that involves multitasking demands. The results indicate that during tRNS, and immediately after stimulation, the performance of the main task was not enhanced by tRNS over the DLPFC: Both groups performed the task at a comparable level immediately. However, at this time scale the stimulation enhanced the performance of the secondary task. Subjects who received active tRNS over the DLPFC showed a significantly higher execution rate of the secondary task immediately after training compared to subjects with sham stimulation. These results are in line with other forms of brain stimulation such as transcranial direct current stimulation, or alternating current stimulation, indicating the causal role of the DLPFC in multitasking (Berryhill et al. 2012, Filmer et al. 2013, Martin et al. 2013, Nelson et al. 2016, Strobach et al. 2013). In the current study, this effect might be related to the requirement to produce a maximum amount of purified waste water at the end of skill 
acquisition (primary task), thus making the secondary task more sensitive to detect effects. This interpretation is compatible with other theoretical frameworks in cognitive psychology, in which the effects of the experimental manipulation are observed in measures that the subjects put less emphasis on (Pachella 1974). For example, in a simple behavioural task, putting more emphasis on reaction times, would lead to greater sensitivity to detect the effect of a given cognitive manipulation on accuracy rather than reaction times. It should also be noted that the lack of tRNS effect on the primary task cannot be attributed easily to a ceiling effect, as the participants demonstrated an increase performance with time.

In contrast, to these immediate and short-term effects, two weeks later those who originally received tRNS showed better retention of the acquired skill compared to those who received sham stimulation, as indicated by better performance in the primary task only. This effect indicates that tRNS is beneficial in retaining the skill in spite of its disuse. However, we also show that the effect depends on general mental ability. Only those with lower general mental ability benefited from tRNS. This observation is in line with previous studies in the field of brain stimulation, which observed beneficial effects of brain stimulation for those with lower cognitive abilities (Santarnecchi et al. 2015, Sarkar, Dowker, and Cohen Kadosh 2014, Looi et al. 2016, Tseng and al. 2012).

The observation that the effects of tRNS were different for low and higher performers is compatible with suggestions that stochastic facilitation may be a mechanism through which tRNS achieves its effects on functioning (van der Groen and Wenderoth 2016, Fertonani and Miniussi 2016). Based on this reasoning, low compared to high performers may have a greater preponderance of neurons that are not reaching their threshold to fire (i.e. subthreshold signals) and thus the quality of their signal output can benefit from the increased levels of unpredictable fluctuations provided 
through tRNS. However, as the effect of other non-tRNS tES protocols have shown similar effects in other cognitive domains, another possibility is that the current effect is not due to stochastic facilitation, but due to other factors such as a ceiling effect in those with higher cognitive abilities.

The effect of tRNS on retention is remarkable, given that its application was for a relatively short period of time compared to most tRNS protocols that aimed to improve skill acquisition (Fertonani, Pirulli, and Miniussi 2011, Looi et al. 2016, Cappelletti et al. 2013, Snowball et al. 2013, Popescu et al. 2016). Such an effect highlights the potential of tRNS as an enhancement method for better skill consolidation.

The effect of tRNS on the secondary task during the immediate and short-term period, while revealing its effect on the main task only two weeks later, suggest that tRNS reduced the cognitive load during the skill acquisition stage. This can also explain the better retention after two weeks. However, another possibility is that such effects are sleep consolidation-related. This idea fits with a recent study showing that tRNS over the DLPFC improved mathematical learning 24 hours after its application, but not immediately (Cohen Kadosh, submitted). Such a dissociation between immediate and delayed effect is not uncommon in the field of cognitive psychology and has been shown in different cognitive domains (for an integrated review see: Soderstrom and Bjork 2015).

The effect of tRNS on the secondary task was observed immediately. This is in contrast to previous studies that have shown that in the first five minutes tRNS does not affect neural excitability and behaviour in a significant manner (Chaieb, Antal, and Paulus 2011, Snowball et al. 2013). It is important to note that the observed immediate effect cannot be easily explained by spurious group differences. It is not possible to 
entirely exclude the possibility of a priori groups differences, as we did not examine the performance on the task to avoid neurostimulation state-dependent effects (Romei, Thut, and Silvanto 2016). However, we would like to emphasise that a few factors should mitigate such concerns. First, all subjects were beginners and had no experience or history with a waste water treatment task. Second, the group differences that we observed at the beginning of task when tRNS applied, were not found when the participants were tested 2 weeks later. This contrasts with what one would expect if the immediate effects were due to a priori groups differences.

As findings accumulate that highlight the potential value of brain stimulation for the field of ergonomics, it will be important for practitioners to consider the associated ethical implications of cognitive enhancement through brain stimulation. On the one hand, one may consider that, in the context of the present findings, the observation that tRNS-related gains in performance were primarily limited to those with lower general mental ability could have ethically favourable implications. Specifically, this may be viewed as ethically favourable for the potential to foster a more egalitarian workforce. However, the ethical implications of brain stimulation are a complex and controversial matter, and we urge the interested reader to refer to previous discussion on this topic (e.g., Cohen Kadosh et al. 2012; Hamilton, Messing, and Chatterjee 2011). At the very least, further work is needed in larger and more heterogenous samples to have a better understanding of its efficacy and safety.

To summarise, our results indicate that tRNS may be a viable tool for enhancing the capacity to multitask in the short-term, with associated longer-term gains in retention, conceivably afforded by a reduction in multitasking costs during initial skill acquisition. The potential application of these findings is exciting, but before 
implementing this approach in real-world occupational settings, future work will be required to establish the efficacy of this approach and the extent to which the findings generalise to other tasks that require multitasking in the context of skill acquisition.

\section{Acknowledgement}

We would like to thank the reviewers for their thoughtful comments and efforts. Further, we thank the team of the Cognition, Learning \& Plasticity lab for their support and meaningful discussions. This work has been supported by the European Research Council [Learning\&Achievement 338065].

\section{References}

Aiken, L. S., and S. G. West. 1991. Multiple regression: Testing and interpreting interactions. Newbury Park: Sage.

Anderson, J.R. 2005. Cognitive psychology and its implications (6. Edition). New York: Worth Pub.

Arthur, W., E.A. Day, W. Bennett, and A.M. Portrey. 2013. Individual and team skill decay: The science and implications for practice. Hove: Routledge.

Berryhill, M.E., E.B. Wencil, H. Branch Coslett, and I.R. Olson. 2012. "A selective working memory impairment after transcranial direct current stimulation to the right parietal lobe." Neurosci. Lett. 479 (3):312-316.

Bjork, R. A., and E. L. Bjork. 1992. "A new theory of disuse and an old theory of stimulus fluctuation." In From learning processes to cognitive processes: Essays in honor of William K. Estes, edited by A. Healy, S. Kosslyn and R. Shiffrin, 3567. Hillsdale: Erlbaum.

Bjork, R. A., and E. L. Bjork. 2006. "Optimizing treatment and instruction: Implications of a new theory of disuse." In Memory and society. Psychological perspectives, edited by L. G. Nilsson and N. Ohta, 116-140. New York: Psychology Press.

Burkolter, D., A. Kluge, S. German, and B. Grauel. 2009. "Waste Water Treatment Simulation (WaTrSim): Validation of a new process control simulation tool for experimental training research." Proceedings of the Human Factors and Ergonomics Society Annual Meeting 53 (26):1969-1973. 
Cappelletti, M., E. Gessaroli, R. Hithersay, M. Mitolo, D. Didino, R. Kanai, R. Cohen Kadosh, and V. Walsh. 2013. "Transfer of Cognitive Training across Magnitude Dimensions Achieved with Concurrent Brain Stimulation of the Parietal Lobe." The Journal of Neuroscience 33 (37):14899-14907.

Chaieb, L., A. Antal, and W. Paulus. 2011. "Transcranial alternating current stimulation in the low kHz range increases motor cortex excitability." Restorative Neurology and Neuroscience 29 (3):167-175.

Chein, J. M., and W. Schneider. 2012. "The Brain's Learning and Control Architecture." Current directions in psychological science 21 (2):78-84.

Cohen Kadosh, R., Levy, N., O'Shea, J., Shea, N., \& Savulescu, J. (2012). “The neuroethics of non-invasive brain stimulation”. Current Biology, 22, R108R111.

Diamond, A. 2013. "Executive Functions." Annual Review of Psychology 64 (1):135168.

Driskell, J. E., R.P. Willis, and C. Copper. 1992. "Effect of overlearning on retention." Journal of Applied Psychology 77 (5):615-622.

Fertonani, A., and C. Miniussi. 2016. "Transcranial Electrical Stimulation:What We Know and Do Not KnowAbout Mechanisms." The Neuroscientist:1-15.

Fertonani, A., C. Pirulli, and C. Miniussi. 2011. "Random Noise Stimulation Improves Neuroplasticity in Perceptual Learning." The Journal of Neuroscience 31 (43):15416-15423.

Filmer, H. L., Mattingley, J. B., and Dux, P. E. (2013). "Improved multitasking following prefrontal tDCS." Cortex, 49 (10), 2845-2852.

Frank, B., and A. Kluge. 2017. "Cued recall with gaze guiding — reduction of human errors with a gaze-guiding tool." In Advances in neuroergonomics and cognitive engineering, edited by K.S. Hale and K.M. Stanney, 3-16. Heidelberg: Springer.

Frank, B., and A. Kluge. 2018. "Complex cognitive skill retention: The roles of general mental ability and refresher interventions in a simulated vocational setting." Journal of Computer Assisted Learning, https://doi.org/10.1111/jcal.12251.

Hamilton, R., Messing, S., \& Chatterjee, A. (2011). "Rethinking the thinking cap". Neurology, 76(2), 187-193. doi:10.1212/WNL.0b013e318205d50d.

Hawkins, K. A., S.V. Faraone, J.R. Pepple, L. J. Seidman, and M.T. Tsuang. 1990. "WAIS — R validation of the Wonderlic Personnel Test as a brief intelligence 
measure in a psychiatric sample." Psychological Assessment: A Journal of Consulting and Clinical Psychology 2 (2):198.

Hayes, A. F. 2013. Introduction to mediation, moderation, and conditional analysis. New York, NY: The Guilford Press.

Hoddes, E., V. Zarcone, H. Smythe, R. Philipp, and W.C. Dement. 1973. "Quantification of Sleepiness: A New Approach." Psychophysiology 10 (4):431436.

Hsu, W.-Y., Zanto, T. P., van Schouwenburg, M. R., and Gazzaley, A. (2017). "Enhancement of multitasking performance and neural oscillations by transcranial alternating current stimulation". PLOS one, https://doi.org/10.1371/journal.pone.0178579

Kluge, A. 2014. The Acquisition of Knowledge and Skills for Taskwork and Teamwork to Control Complex Technical Systems. Heidelberg: Springer.

Kluge, A., and B. Frank. 2014. "Counteracting skill decay: four refresher interventions and their effect on skill and knowledge retention in a simulated process control task." Ergonomics 57 (2):175-190.

Krause, B., and R. Cohen Kadosh. 2013. "Can transcranial electrical stimulation improve learning difficulties in atypical brain development? A future possibility for cognitive training." Developmental Cognitive Neuroscience 6 (100):176-194.

Looi, C. Y., M. Duta, A. K. Brem, S. Huber, H.K. Nuerk, and R. Kohen Cadosh. 2016. "Combining brain stimulation and video game to promote long-term transfer of learning and cognitive enhancement." Scientific Reports 6:22003.

Martin, D.M., R. Liu, A. Alonzo, M. Green, M.J. Player, P. Sachdev, and C.K. Loo. 2013. "Can transcranial direct current stimulation enhance outcomes from cognitive training? A randomized controlled trial in healthy participants." Int J Neuropsychopharmacol 16 (9):1927-1936.

McDaniel, M.A., P.C. Brown, and H.L. Roediger. 2014. Make it stick. Cambridge: Harvard University Pres.

McDonnell, M. D., and L. M. Ward. 2011. "The benefits of noise in neural systems: bridging theory and experiment." Nature Reviews Neuroscience 12 (415).

Nelons, J., McKinley, R. A., Philipps, C., McIntire, L., Goodyear, C., Kreiner, A., and Monforten, A. (2016). "The Effects of Transcranial Direct Current Stimulation (tDCS) on Multitasking Throughput Capacity". Front Hum Neurosci, https://doi.org/10.3389/fnhum.2016.00589. 
Pachella, R. 1974. "The interpretation of reaction time in information processing research." In Human information processing: Tutorials in performance and cognition, edited by B. H. Kantowitz, 41-82. Hillsdale, NJ: Erlbaum.

Parasuraman, R. 2003. "Neuroergonomics: Research and practice." Theoretical Issues in Ergonomics Science 4 (1-2):5-20.

Popescu, T., B. Krause, D. B. Terhune, O. Twose, T. Page, G. Humphreys, and R. Cohen Kadosh. 2016. "Transcranial random noise stimulation mitigates increased difficulty in an arithmetic learning task." Neuropsychologia 81:255264.

Proctor, R. W., and A. Dutta. 1995. Skill acquisition and human performance. Thousand Oaks: Sage Publications, Inc.

Romei, V., Thut, G., and Silvanto, J. (2016). “Information-Based Approaches of Noninvasive Transcranial Brain Stimulation”. Trends Neurosci, 39(11), 782795. doi:10.1016/j.tins.2016.09.001

Santarnecchi, E., A.K. Brem, E. Levenbaum, T. Thompson, R. Cohen Kadosh, and A. Pascual-Leone. 2015. "Enhancing cognition using transcranial electrical stimulation." Curr.Opin.Behav.Sci. 4:171-178.

Sarkar, A., A. Dowker, and R. Cohen Kadosh. 2014. "Cognitive Enhancement or Cognitive Cost: Trait-Specific Outcomes of Brain Stimulation in the Case of Mathematics Anxiety." The Journal of Neuroscience 34:16605-16610.

Schroeder, P. A., T. Dresler, J. Bahnmueller, C. Artemenko, R. Cohen Kadosh, and H.C. Nuerk. 2017. "Cognitive Enhancement of Numerical and Arithmetic Capabilities: A Mini-Review of Available Transcranial Electric Stimulation Studies." Journal of Cognitive Enhancement 1 (1):39-47.

Simonsmeier, B. A., R. H. Grabner, J. Hein, U. Krenz, and M. Schneider. 2018. "Electrical brain stimulation (tES) improves learning more than performance: A meta-analysis." Neurosci Biobehav Rev 84:171-181.

Snowball, A., I. Tachtsidis, T. Popescu, J. Thompson, M. Delazer, L. Zamarian, T. Zhu, and R. Cohen Kadosh. 2013. "Long-term enhancement of brain function and cognition using cognitive training and brain stimulation." Current Biology 23 (11):987-992.

Soderstrom, N. C., and R. A. Bjork. 2015. "Learning Versus Performance: An Integrative Review." Perspectives on Psychological Science 10 (2):176-199. 
Strobach, T., R. Liepelt, H Pashler, P. A. Frensch, and T. Schubert. 2013. "Effects of extensive dual-task practice on processing stages in simultaneous choice tasks." Attention, Perception, \& Psychophysics 75 (5):900-920.

Terney, D., L. Chaieb, V. Moliadze, A. Antal, and W. Paulus. 2008. "Increasing human brain excitability by transcranial high-frequencyrandom noise stimulation." Journal of Neurosciene 28 (52):14147-14155.

Tseng, P., and et al. 2012. "Unleashing Potential: Transcranial Direct Current Stimulation over the Right Posterior Parietal Cortex Improves Change Detection in Low-Performing Individuals." The Journal of Neuroscience 32:10554-10561. van der Groen, O., and N. Wenderoth. 2016. "Transcranial Random Noise Stimulation of Visual Cortex: Stochastic Resonance Enhances Central Mechanisms of Perception." The Journal of Neuroscience 36 (19):5289-5298.

van Merriënboer, J. J.G. 1997. Training complex cognitive skills: A four-component instructional design model for technical training. Englewood Cliffs: Educational Technology.

Wickens, C. D. 2008. "Situation awareness: review of Mica Endsley's 1995 articles on situation awareness theory and measurement." Human factors 50 (3):397-403.

Wickens, C. D., and J. G. Hollands. 2000. Engineering psychology and human performance. Vol. 3. Upper Saddle River: Prentice Hall.

Wickens, C. D., and J. S. McCarley. 2008. Applied attention theory. Boca Raton: CRC Press.

Wonderlic, Inc. 2002. Wonderlic Personnel Test. Libertyville: Wonderlic Inc. 
Table 1. Description of main and secondary task of WaTrSim operation. Main task is operated step by step, and secondary task is handled every 50 seconds.

\begin{tabular}{|c|c|c|c|}
\hline Step & $\begin{array}{l}\text { Main task } \\
\text { Task: Follow start-up procedure } \\
\text { Objective: Production outcome }\end{array}$ & Seconds & $\begin{array}{l}\text { Secondary Task } \\
\text { Task: Monitor tank level of tank Ba every } 50 \\
\text { seconds } \\
\text { Objective: Monitoring }\end{array}$ \\
\hline 1 & LIC V9: Flow rate $500 \mathrm{l} / \mathrm{h}$ & $0-49$ & Monitor tank level of tank $\mathrm{Ba}$ \\
\hline 2 & V2 deactivate follower control & 50 & $\begin{array}{l}\text { Report approximate tank level of tank Ba: } \\
\text { Click on button Monitoring and set number of } \\
\text { tank level }\end{array}$ \\
\hline 3 & Valve V1: Flow rate $500 \mathrm{l} / \mathrm{h}$ & $51-99$ & Monitor tank level of tank Ba \\
\hline 4 & Wait until R1 > 2001 & 100 & $\begin{array}{l}\text { Report approximate tank level of tank Ba: } \\
\text { Click on button Monitoring and set number of } \\
\text { tank level }\end{array}$ \\
\hline 5 & Valve V2: Flow rate 500 1/h & 101-149 & Monitor tank level of tank Ba \\
\hline 6 & Wait until R1 > 4001 & 150 & $\begin{array}{l}\text { Report approximate tank level of tank Ba: } \\
\text { Click on button Monitoring and set number of } \\
\text { tank level }\end{array}$ \\
\hline 7 & Valve V3: Flow rate 1000 1/h & $151-180$ & - \\
\hline 8 & Wait until HB1 > 1001 & & \\
\hline 9 & Activate heating HB1 & & \\
\hline 10 & Wait until $\mathrm{HB} 1>60^{\circ} \mathrm{C}$ & & \\
\hline 11 & Activate column K1 & & \\
\hline 12 & Valve V4: Flow rate 1000 1/h & & \\
\hline 13 & Valve V6: Flow rate 400 1/h & & \\
\hline
\end{tabular}


$\underline{\text { Table 2. Performance of active and sham group }}$

active group $(n=20)$

sham group $(n=19)$

Main task

Production outcome (litres) - Training $1 \quad 173.50(86.69,0-331.01) \quad 155.39(74.91,0-253.01)$

Production outcome (litres) - Training 2274.35 (82.48, 75.505-385.01) 230.30 (73.62, 57.51-321.01)

Production outcome (litres) - Test $1 \quad 283.47(83.26,101.80-430.39) \quad 271.81(73.50,113.86-347.01)$

Production outcome (litres) - Test $2 \quad 114.02(117.35,0-407.01) \quad 67.31(100.96,0-300.01)$

Secondary task

Monitoring (0-3) - Training 1

$1.00(0.86,0-3.00)$

$0.13(0.40,0-1.50)$

Monitoring (0-3) - Training 2

$1.75(1.15,0-3.00)$

$0.40(0.86,0-3.00)$

Monitoring (0-3) - Test 1

$1.93(1.04,0-3.00)$

$0.74(1.21,0-3.00)$

Monitoring (0-3) - Test 2

$0.55(0.83,0-2.50)$

$0.26(0.56,0-1.50)$

Note: $N=39$; Performance of both groups was measured for the first six minutes of training (Training 1), the latter six minutes of training (Training 2) and for nine minutes of Test 1 at skill acquisition. After two weeks, performance was measured for six minutes of Test 2 at recall; $M$ $(S D$, range) 